\title{
Spectral Velocity Estimation in the Transverse Direction
}

\author{
Jensen, Jørgen Arendt
}

Published in:

Proceedings of IEEE International Ultrasonics Symposium

Link to article, DOI:

10.1109/ULTSYM.2013.0163

Publication date:

2013

Document Version

Early version, also known as pre-print

Link back to DTU Orbit

Citation (APA):

Jensen, J. A. (2013). Spectral Velocity Estimation in the Transverse Direction. In Proceedings of IEEE International Ultrasonics Symposium (pp. 631-634). IEEE. https://doi.org/10.1109/ULTSYM.2013.0163

\section{General rights}

Copyright and moral rights for the publications made accessible in the public portal are retained by the authors and/or other copyright owners and it is a condition of accessing publications that users recognise and abide by the legal requirements associated with these rights.

- Users may download and print one copy of any publication from the public portal for the purpose of private study or research.

- You may not further distribute the material or use it for any profit-making activity or commercial gain

- You may freely distribute the URL identifying the publication in the public portal

If you believe that this document breaches copyright please contact us providing details, and we will remove access to the work immediately and investigate your claim 
Paper presented at the IEEE International Ultrasonics Symposium, Prague, Czech Republic, 2013:

\title{
Spectral Velocity Estimation in the Transverse Direction
}

\author{
Jørgen Arendt Jensen
}

Center for Fast Ultrasound Imaging,

Biomedical Engineering group, Department of Electrical Engineering, Bldg. 349, Technical University of Denmark, DK-2800 Kgs. Lyngby, Denmark 


\title{
Spectral Velocity Estimation in the Transverse Direction
}

\author{
Jørgen Arendt Jensen \\ Center for Fast Ultrasound Imaging, Department of Electrical Engineering, \\ Technical University of Denmark, DK-2800 Lyngby, Denmark
}

\begin{abstract}
A method for estimating the velocity spectrum for a fully transverse flow at a beam-to-flow angle of $90^{\circ}$ is described. The approach is based on the Transverse Oscillation (TO) method, where an oscillation across the ultrasound beam is made during receive processing. A fourth-order estimator based on the correlation of the received signal is derived. A Fourier transform of the correlation signal yields the velocity spectrum. Performing the estimation for short data segments gives the velocity spectrum as a function of time as for ordinary spectrograms, and it also works for a beam-to-flow angle of $90^{\circ}$. The approach is validated using Field II simulations. A $3 \mathrm{MHz}$ convex array with lambda pitch is modeled. The transmit focus is at 200 mm and 2 times 32 elements are used in receive. A dual-peak Hamming apodization with a spacing of 96 elements between the peaks is used during receive beamforming for creating the lateral oscillation. Pulsatile flow in a femoral artery placed 40 $\mathrm{mm}$ from the transducer is simulated for one cardiac cycle using the Womersly-Evan's flow model. The bias of the mean estimated frequency is $13.6 \%$ compared to the true velocity and the mean relative std is $14.3 \%$. This indicates that the new estimation scheme can reliably find the spectrum at $90^{\circ}$, where a traditional estimator yields zero velocity. Measurements have been conducted with the SARUS experimental scanner and a BK 8820e convex array transducer (BK Medical, Herlev, Denmark). A CompuFlow 1000 (Shelley Automation, Inc, Toronto, Canada) generated the artificial femoral artery flow in the phantom. It is demonstrated that the transverse spectrum can be found from the measured data. The estimated spectra degrade when the angle is different from $90^{\circ}$, but are usable down to $60-70^{\circ}$. Below this angle the traditional spectrum is best and should be used. The conventional approach can automatically be corrected for angles from $0-70^{\circ}$ to give a fully quantitative velocity spectrum without operator intervention.
\end{abstract}

\section{INTRODUCTION}

Traditional ultrasound scanners can display the velocity distribution at one given depth in a vessel as a function of time in a spectrogram display. The spectrogram is calculated by measuring the sampled signal at the chosen depth and then employing a short time Fourier transform on the received data [1], [2], [3]. The spectra are then stacked side-by-side to show the time evolution of the velocity distribution as shown in Fig. 1.

The relation between the blood velocity and the frequency measured $f_{p}$ is given by:

$$
f_{p}=\frac{2 v_{z}}{c} f_{0}=\frac{2|\vec{v}| \cos \Theta}{c} f_{0}
$$

where $f_{0}$ is the emitted ultrasound frequency, $c$ is the speed of sound and $v_{z}$ is the blood velocity in the axial direction. $\Theta$ is

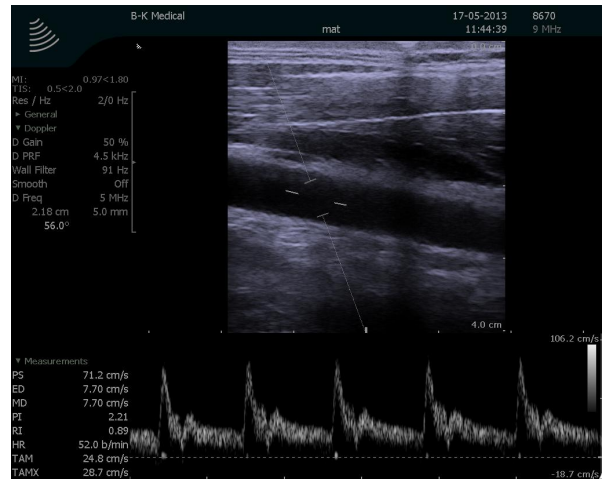

Fig. 1. Duplex ultrasound image of the carotid artery. On the top is the B-mode image showing the anatomy and the placement of the measurement region (range gate) indicated by the broken line. The lower graph shows the velocity spectrum as a function of time.

the angle between the blood velocity vector and the ultrasound beam, and only the projected velocity component is measured by these systems. The measurements need to be corrected for the beam-to-flow angle, and at $90^{\circ}$ no velocity is found. This is a major problem, since the vessels often are perpendicular to the ultrasound beam direction, and angles above $70^{\circ}$ can lead to large estimation errors for small angle deviations. It is therefore advisable to keep the angle below $60^{\circ}$ for accurate results.

The problem can be solved by introducing an oscillation transverse to the ultrasound beam, so that the measured signal is sensitive to a transverse motion. This will yield signals with an oscillation frequency proportional to the transverse velocity. This paper describes a new method for making spectral velocity estimation in the direction transverse to the ultrasound propagation direction. Two estimators are derived: a second order and a fourth-order, Both are based on the Transverse Oscillation (TO) approach previously used for vector flow estimation. Both estimators are evaluated using Field II simulations in Section III for pulsatile flow in the femoral artery. The approach has also been implemented on the SARUS experimental scanner, and results from a pulsatile flow experiment are shown in Section IV.

\section{Spectral Velocity Estimator}

The power density spectrum of the received signal is found using the TO approach [4], [5], where a transverse oscillation 
is introduced during the receive beamforming. Two image lines are focused in parallel, where the left one contains the in-phase and the right the quadrature component. This complex signal can, at one fixed depth, be described by [6]:

$$
r_{s q}(i)=\cos \left(2 \pi f_{p} i T_{p r f}\right) \exp \left(j 2 \pi f_{x} i T_{p r f}\right),
$$

where $i$ is the emission number, $T_{p r f}$ is the pulse repetition period and $f_{p}$ is the received axial frequency given by (1). $f_{x}$ is the transverse oscillation frequency and is equal to to $v_{x} / \lambda_{x}$, where $\lambda_{x}$ is the transverse oscillation period and $v_{x}$ the transverse velocity. The temporal Hilbert transform of (2) is

$$
r_{s q h}(i)=\sin \left(2 \pi f_{p} i T_{p r f}\right) \exp \left(j 2 \pi f_{x} i T_{p r f}\right) .
$$

Combining (2) and (3) and using Euler's equations gives

$$
\begin{array}{r}
r_{s q}(i)=\frac{1}{2}\left(\exp \left(j 2 \pi i T_{p r f}\left(f_{x}+f_{p}\right)\right)+\exp \left(j 2 \pi i T_{p r f}\left(f_{x}-f_{p}\right)\right)\right) \\
r_{s q h}(i)=\frac{1}{2 j}\left(\exp \left(j 2 \pi i T_{p r f}\left(f_{x}+f_{p}\right)\right)-\exp \left(j 2 \pi i T_{p r f}\left(f_{x}-f_{p}\right)\right)\right) .
\end{array}
$$

Two new signals are then formed from:

$$
\begin{aligned}
& r_{1}(i)=r_{s q}(i)+j r_{s q h}(i)=\exp \left(j 2 \pi i T_{p r f}\left(f_{x}+f_{p}\right)\right) \\
& r_{2}(i)=r_{s q}(i)-j r_{s q h}(i)=\exp \left(j 2 \pi i T_{p r f}\left(f_{x}-f_{p}\right)\right) .
\end{aligned}
$$

The power density spectrum of a stochastic signal is found from:

$$
P_{11}(f)=\sum_{k=-\infty}^{+\infty} R_{11}(k) \exp (-j 2 \pi f k),
$$

where $R_{11}(k)$ is the autocorrelation of the received signal. For the transverse velocity component this can be derived from the cross-correlation function $R_{12}(k)$ between the spatial in-phase and quadrature signal given as:

$$
\begin{aligned}
R_{12}(k)= & \sum_{i=-\infty}^{+\infty} r_{1}(i) r_{2}(i+k) \\
= & \sum_{i=-\infty}^{+\infty} \exp \left(j 2 \pi i T_{p r f}\left(f_{x}+f_{p}\right)\right) \\
& \quad \times \exp \left(j 2 \pi(i+k) T_{p r f}\left(f_{x}-f_{p}\right)\right) \\
= & \exp \left(j 2 \pi k T_{p r f}\left(f_{x}-f_{p}\right)\right) \sum_{i=-\infty}^{+\infty} \exp \left(j 2 \pi i T_{p r f} 2 f_{x}\right) \\
= & \exp \left(j 2 \pi k T_{p r f} f_{p}\right) \times \quad \sum_{i=-\infty}^{+\infty} \exp \left(j 2 \pi k(i+k) T_{p r f} f_{x}\right) \exp \left(j 2 \pi i T_{p r f} f_{x}\right) \\
= & \exp \left(-j 2 \pi k T_{p r f} f_{p}\right) R_{11}(k) .
\end{aligned}
$$

The frequency $f_{p}$ is zero in the case there is no axial velocity component $\left(v_{z}=0\right)$ and the cross-correlation directly equals the autocorrelation of the transverse signal. Making a short Fourier transform of this, thus, directly reveals the transverse velocity spectrum. This is called the second order estimator.

The compensation for the axial velocity can also be made using a fourth-order estimator similar to the one derived in
[6]. The fourth order correlation function is calculated as:

$$
\begin{aligned}
& R_{11}(k)=\sum_{i=-\infty}^{+\infty} r_{1}(i) r_{1}^{*}(i+k), \\
& R_{22}(k)=\sum_{i=-\infty}^{+\infty} r_{2}(i) r_{2}^{*}(i+k) \\
& R_{44}(k)=R_{11}(k) \cdot R_{22}(k),
\end{aligned}
$$

which eliminates the axial component. An elimination of the lateral component can be done using:

$$
R_{44 a x}(k)=R_{11}(k) \cdot R_{22}^{*}(k) .
$$

The spectrum is then found from:

$$
P_{44}(f)=\sum_{k=-\infty}^{+\infty} R_{44}(k) \exp (-j 2 \pi f k) .
$$

This approach is called the fourth-order method.

\section{Simulation of TO Data And Results}

Blood flow in the femoral artery has been simulated using Field II [7], [8] and the Womersley-Evans' pulsatile flow model [9], [2]. The model gives realistic simulated velocity profiles for a normal human geometry [10]. A $3 \mathrm{MHz}$ convex array transducer with 128 active elements has been used. The center 64 elements are used in transmit, and the outer 64 elements are used during receive. The TO field has been optimized for imaging at $40 \mathrm{~mm}$ by adjusting the time delays and apodization function [11].

The resulting spectra for the second order method is shown in Fig. 2 for a beam-to-flow angle of $90^{\circ}$ (top) and $60^{\circ}$ (bottom). The solid green curve shows the peak velocity at the center of the vessel. The spectrum can be estimated at $90^{\circ}$ with some bias, but both positive and negative velocities are shown. At $60^{\circ}$ the modulation from the term $\exp \left(-j 2 \pi k T_{p r f} f_{p}\right)$ in (7) sets in and severely distorts the spectrum, thus, making is useless for quantitative studies.

The resulting power density spectrograms for different angles for the fourth-order methods are shown in Fig. 3. It is seen that the spectrogram can reliably be estimated for fully transverse flow. The estimates get progressively worse when the beam-to-flow angle deviates from $90^{\circ}$ as shown in Fig. 4 for $60^{\circ}$. The transverse velocity spectra is considerably widened and gets less accurate. This is not a major problem as the axial velocity spectrum now is a reliable measure of velocity distribution. For angles lower than $60^{\circ}$ the normal spectrogram can be used and gives a good estimate. The angle can easily be estimated from the TO data measured for the spectral estimation and be used for selecting the spectrum to display. It can also automatically adjust the velocity axis for the axial velocity spectrum.

The mean velocity for the fourth-order spectrum has been calculated and is shown as the blue curve in Fig. 5 as a function of time. The corresponding true mean velocity is shown also. The bias relative to peak velocity in the vessel is $13.6 \%(0.18$ $\mathrm{m} / \mathrm{s}$ ) and the relative std. is $0.19 \mathrm{~m} / \mathrm{s}(14.2 \%)$. 

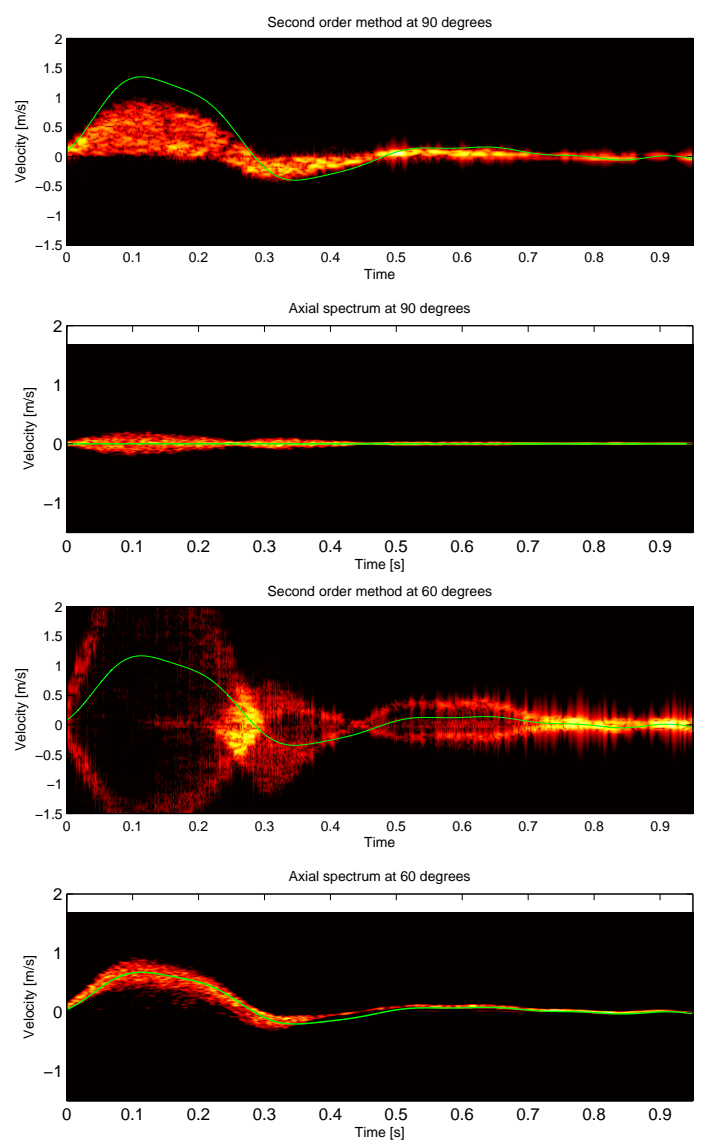

Fig. 2. Estimated power density spectrograms for the second order method for a simulated femoral artery. The lateral (top) and axial velocities (bottom) are shown for $90^{\circ}$ (top) and $60^{\circ}$ (bottom) flow angles.

\section{Flow Rig Measurements}

Measurements have been made using the SARUS experimental ultrasound scanner [12] connected to a BK 8820e (BK Medical, Herlev, Denmark) convex array probe. A duplex sequence was employed with B-mode and TO emissions interleaved with a pulse repetition frequency of $5 \mathrm{kHz}$. The flow lines were emitted with the center of the aperture and focused straight down. The probe was operated at $3 \mathrm{MHz}$ and 64 elements was during transmit that was focused at $105 \mathrm{~mm}$ (F\# of 5). The received signal of all 192 transducer elements was sampled at $17.5 \mathrm{MHz}$ with 12 bits precision. The two TO beams were focused using the center 96 elements and two von Hann apodization peaks each with a width of 32 elements and their peaks separated by 64 elements. The lateral wavelength $\lambda_{x}$ was set to $0.88 \mathrm{~mm}$ for the focusing.

The flow was generated by a CompuFlow 1000 (Shelley Automation, Inc, Toronto, Canada) connected to a dedicated carotid bifurcation phantom (Danish Phantom Design, Frederikssund, Denmark). filled with Shelley Blood Mimicking Fluid for ultrasound (BMF-3L-US, Shelley Automation, Inc, Toronto, Canada). The $8 \mathrm{~mm}$ diameter straight tube entrance to the bifurcation was used as the measurement site. The pump was set to use the femoral waveform to mimic the waveform found in the femoral artery with both positive and negative
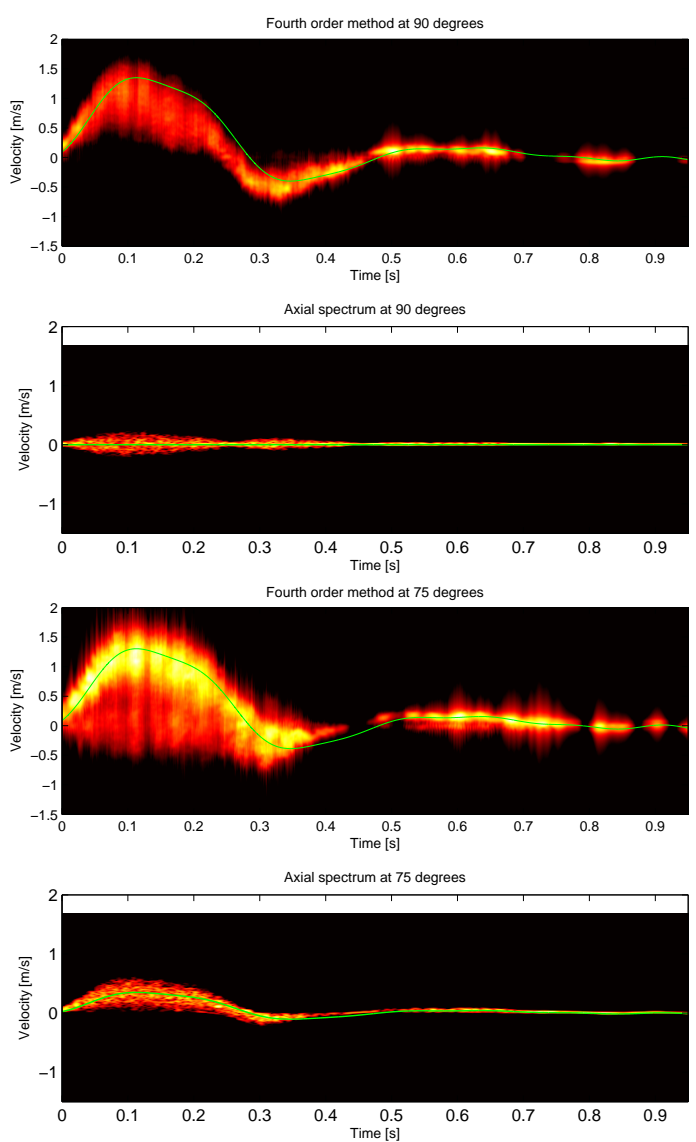

Fig. 3. Estimated power density spectrograms for the fourth-order method for a simulated femoral artery. The lateral (top) and axial velocities (bottom) are shown for $90^{\circ}$ (top) and $75^{\circ}$ (bottom) flow angles.
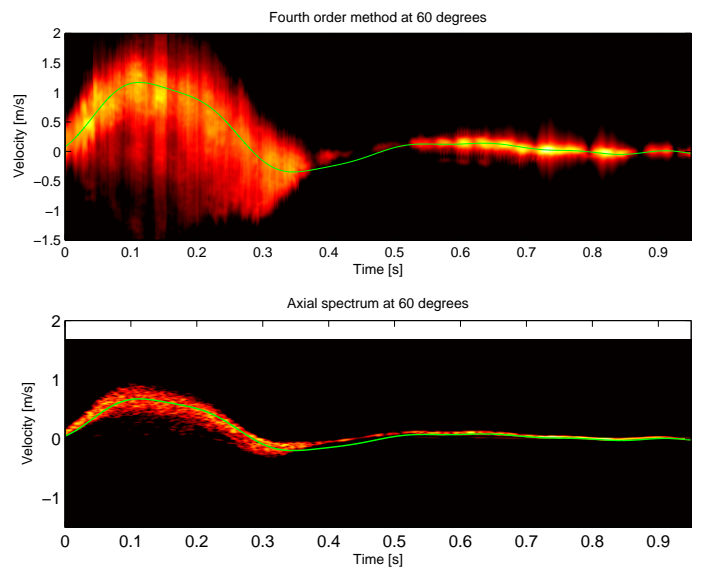

Fig. 4. Estimated power density spectrograms for the fourth-order method for a simulated femoral artery. The lateral (top) and axial velocities (bottom) are shown for a $60^{\circ}$ flow angle. 


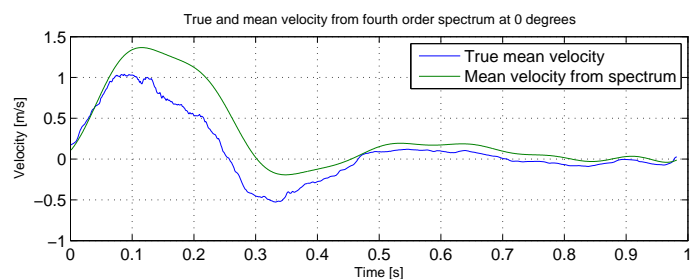

Fig. 5. Estimated mean velocity for fourth-order spectrum compared to true mean velocity for $90^{\circ}$.

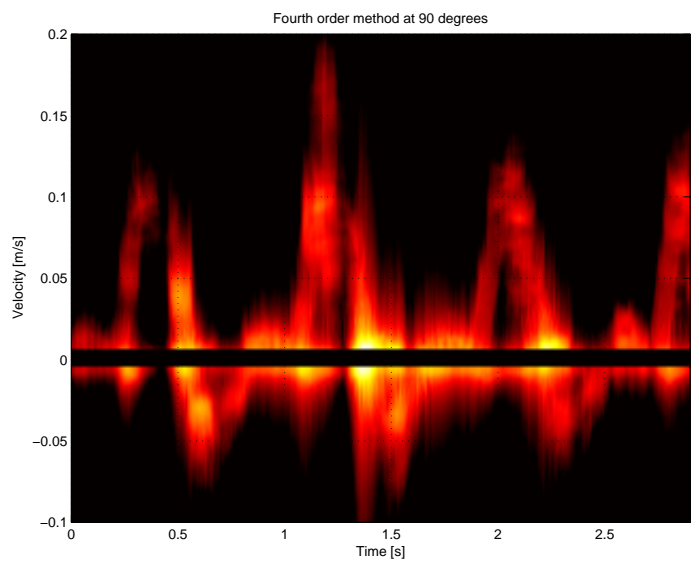

Fig. 6. Measured fourth-order spectrum for pulsatile flow mimicking that found in the femoral artery.

velocities. The peak volume flow was set to $10 \mathrm{~mL} / \mathrm{s}$, and the beam to flow angle was estimated to be $91.0^{\circ} \pm 3.3^{\circ}$ from the velocity data.

Data were acquired continuously for 3 seconds yielding 3.8 Gbytes of fully sampled RF data. It was beamformed and the fourth-order estimator was used on the focused TO data. The resulting fourth-order spectrum found at the center of the vessel is shown in Fig. 6 for three seconds. Four peaks in the systolic phases can be seen in the data with a positive velocity followed by negative velocities in the diastole as expected. The peak velocity is fairly low due to limitations in the setup.

The velocity has also been estimated using the traditional TO velocity estimators [6], and this is shown as a function of both time and depth in Fig. 7 for the lateral velocity component $v_{x}$. Here the peak velocities agrees with that found by the fourth-order spectral estimator.

\section{Discussion AND CONCLUSION}

A new approach for spectral velocity estimation for a transverse flow has been presented. The estimator can reliably find the spectrum at $90^{\circ}$, where a traditional estimator yields a zero velocity. The spectral estimates degrade when the angle is different from $90^{\circ}$ but are usable down to $60-70^{\circ}$. Below this angle the traditional spectrum estimate is best and should be used. The selection between the two spectra can be based on the estimated angle at the range gate, which can be obtained using the normal TO estimator without additional beamforming.

It should be noted that the velocity range is significantly

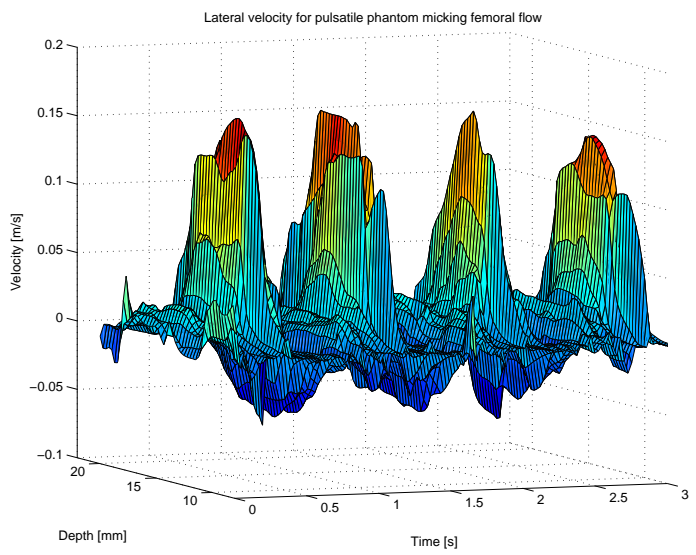

Fig. 7. Transverse velocity component for the pulsatile flow found as a function of time and depth.

higher for the new fourth-order method compared to traditional estimation, as the lateral wavelength is larger than the axial wavelength. That is beneficial for either keeping the pulse repetition frequency low or for maintaining a high maximum detectable velocity.

\section{ACKNOWLEDGEMENT}

This work was supported by grant grant 024-2008-3 from the Danish Advanced Technology Foundation and by B-K Medical Aps.

\section{REFERENCES}

[1] D. W. Baker, "Pulsed ultrasonic Doppler blood-flow sensing," IEEE Trans. Son. Ultrason., vol. SU-17, pp. 170-185, 1970.

[2] D. H. Evans, W. N. McDicken, R. Skidmore, and J. P. Woodcock, Doppler Ultrasound, Physics, Instrumentation, and Clinical Applications. New York: John Wiley \& Sons, 1989.

[3] J. A. Jensen, Estimation of Blood Velocities Using Ultrasound: A Signal Processing Approach. New York: Cambridge University Press, 1996.

[4] J. A. Jensen and P. Munk, "A New Method for Estimation of Velocity Vectors," IEEE Trans. Ultrason., Ferroelec., Freq. Contr, vol. 45, pp. 837-851, 1998.

[5] M. E. Anderson, "Multi-dimensional velocity estimation with ultrasound using spatial quadrature," IEEE Trans. Ultrason., Ferroelec., Freq. Contr., vol. 45, pp. 852-861, 1998.

[6] J. A. Jensen, "A New Estimator for Vector Velocity Estimation," IEEE Trans. Ultrason., Ferroelec., Freq. Contr., vol. 48, no. 4, pp. 886-894, 2001.

[7] J. A. Jensen and N. B. Svendsen, "Calculation of Pressure Fields from Arbitrarily Shaped, Apodized, and Excited Ultrasound Transducers," IEEE Trans. Ultrason., Ferroelec., Freq. Contr., vol. 39, pp. 262-267, 1992.

[8] J. A. Jensen, "Field: A program for simulating ultrasound systems," Med. Biol. Eng. Comp., vol. 10th Nordic-Baltic Conference on Biomedical Imaging, Vol. 4, Supplement 1, Part 1, pp. 351-353, 1996.

[9] J. R. Womersley, "Oscillatory motion of a viscous liquid in a thin-walled elastic tube. I: The linear approximation for long waves," Phil. Mag., vol. 46, pp. 199-221, 1955.

[10] W. W. Nichols and M. F. O'Rourke, McDonald's Blood Flow in Arteries, Theoretical, Experimental and Clinical Principles. Philadelphia: Lea \& Febiger, 1990.

[11] J. A. Jensen, "Optimization of transverse oscillating fields for vector velocity estimation with convex arrays," in Proc. IEEE Ultrason. Symp., July 2013

[12] J. A. Jensen, H. Holten-Lund, R. T. Nilsson, M. Hansen, U. D. Larsen, R. P. Domsten, B. G. Tomov, M. B. Stuart, S. I. Nikolov, M. J. Pihl, Y. Du, J. H. Rasmussen, and M. F. Rasmussen, "SARUS: A synthetic aperture real-time ultrasound system," IEEE Trans. Ultrason., Ferroelec., Freq. Contr., p. In press, 2013. 